\title{
REMARQUES SUR LE CALCUL ÉCONOMIQUE D'UN SYSTEMME D'AMENÉE D'EAU, D'UNE CONDUITE FORCEE OU D'UN CANAL DE FUITE
}

(Méthode de la Longueur Fictive)

\author{
REMARKS ON THE CALCULATION OF THE ECONOMICS OF A PENSTOCK \\ OR TAIL-RACE WATER SUPPLY SYSTEM
}

par F. HAUSER

Ingénieur des Arts et Manufactures

English synopsis p. 226

\section{I. - PRÉAMBule}

Le lecteur nous accordera que le calcul économique fait partie de l'art de l'ingénieur. II n'est pas, en effet, de projet poussé qui ne doive s'accompagner d'étude des prix. Même dans la phase des avant-projets, la solution que l'on choisira devra d'abord répondre au problème posé, ensuite s'adapter aux possibilités d'exécution, enfin être la plus économique qu'il est possible.

Nous désirons étudier ici, dans le cas particulier mentionné en titre, le problème plus général suivant :

Lorsque l'on s'est fixé le principe d'une solution, le prix de revient global des ouvrages est très souvent la somme des prix de deux ou plusieurs ouvrages, dont les coûts varient en sens inverse lorsque l'on exige d'eux un résultat technique constant. Comment choisir les dimensions les plus économiques correspondant aux données techniques imposées?

Traiter un problème économique c'est évidemment schématiser le problème technique: on ne peut pas toujours dire que le prix d'un ouvrage varie d'une manière continue et nous n'en citerons qu'un exemple: celui du batardeau, ou du barrage, dont on est amené à changer le type si l'on dépasse une certaine cote et dont. en conséquence, le prix varie brutalement à ce moment. Cependant, l'ingénieur peut se permettre des hypothèses simplificatrices, telles que, dans certaines limites, la continuité des prix en fonction des variables qui caractérisent les ouvrages. C'est dans ces limites que nous nous plaçons.

Le problème que nous voulons examiner n'est ni celui du «tracé économique», ni celui du choix d'une dénivelée ou d'une perte de charge d'un système d'amenée ou de fuite (1); c'est celui du choix des dimensions d'une succession de canaux ou galeries ou conduites forcées dont on se donne la perte de charge totale. Pour simplifier, nous n'admettons aucune ramification. aucun "service en route »; le débit est donc unique d'un bout à l'autre du système et c'est pour le débit maximum que l'on s'impose une perte de charge maximum.

Soient $x_{1}, x_{2} \ldots . . x_{n}$. les $n$ paramètres caractérisant $n$ tronçons distincts de notre système d'adduction ou de fuite (que ce système soit homogène, c'est-à-dire composé de $n$ tronçons du même type - exemple $n$ tronçons de conduite forcée - ou hétérogène, c'est-à-dire composé de tronçons différents - exemple: canal en terre puis canal revêtu, ou galerie d'amenée puis conduite forcée, etc...). - Le projet doit être suffisamment avancé pour que les longueurs de chacun de ces tronçons soient connues, ainsi que leur type.

L'expression du prix est de la forme:

$$
P=F\left(x_{1}, x_{2}, \ldots \ldots x_{n}\right)
$$

(1) Nous ne traitons pas ici de cet autre problème, qui est un problème de productivité ou d'estimation de besoins. 
En général, $F$ est la somme de $n$ fonctions simples - ou supposées telles - de chacune des variables, et l'on désire:

$$
P \text { minimum }
$$

D'autre part, on s'impose une perte de charge donnée, donc on s'impose:

$$
G\left(x_{1}, x_{2}, x_{3} \ldots x_{n}\right)=B
$$

En général, $G$ est la somme de $n$ fonctions simples de chacune des variables.

Résoudre le problème posé c'est trouver les valeurs de $x_{1}, x_{2} \ldots x_{n}$ qui satisfont aux condi.. tions (1) et (2).

\section{II. - PROFILS TRANSVERSAUX DES TRONCSONS ET FORMULES DE PRIX}

S'il s'agit de conduites forcées, le profil transversal est évidemment le cercle. Mais si l'on considère des écoulements en canaux ou en galerie en charge ou à surface libre ou en conduites à surface libre, les profils transversaux des ouvrages et les profils mouillés peuvent être infiniment variés. Ils sont essentiellement choisis d'après les circonstances locales et l'on n'en peut dire grand chose à l'avance.

Pour un même tronçon, on est amené à considérer non pas un profil, mais une famille de profils entièrement définis par une grandeur $x$, grandeur que les circonstances locales ou le calcul économique déterminent. Pour certains tronçons cette famille pourra englober tous les profils semblables (exemple : conduites forcées ou galeries en charge, sans difficultés spéciales d'exécution). Pour d'autres tronçons, on peut s'imposer de ne pas changer la profondeur, fixée d'après la cote de la nappe d'eau, et se donner seulement latitude de changer la largeur; pour d'autres, des considérations d'emprises et d'expropriation fixent la largeur, et c'est la profondeur qui peut varier, etc... Dans tous les cas, la surface mouillée, la surface intervenant dans le coût des travaux, et le périmètre mouillé peuvent s'exprimer en fonction de $x$ qui peut être, suivant les cas, une largeur, un diamètre ou un rayon hydraulique.

Le coût du mètre courant d'un tronçon peut, pour de tels calculs économiques, être mis sous la forme:

$$
\begin{aligned}
& P=a S+b X \\
&(S=\text { surface } \\
& X=\text { périmètre })
\end{aligned}
$$

Il y a des cas où le terme en $\mathrm{X}$ est nul ou faible (exemple: canal en terre), d'autres, où il constitue la majeure partie du coût (exemple: canal revêtu sous la nappe phréatique, avec difficultés d'exécution).

S'il s'agit de choisir le profil d'un tronçon parmi des profils dont seule la largeur peut varier, $P$ devient de la forme $a L+b$. Si le profil doit être choisi parmi des profils semblables, $P$ prend la forme $a R^{2}+b R$ ( $R$ rayon hydraulique). Dans certains cas, le terme a $R^{2}$ sera fortement prépondérant; dans d'autres, ce sera le terme bR. Si la prépondérance de l'un ou l'autre terme est moins marquée, on pourra assimiler le plus faible au plus fort: si bR est faible devant $a R^{2}$, $b R^{2}$

on pourra l'écrire $\frac{}{R_{m}}$; si $a R^{2}$ est faible devant $b R$, on pourra l'écrire $a R_{m} . R, R_{m}$ étant la valeur présumée du rayon hydraulique après calcul économique. Ainsi $P$ sera ramené à un polynome du premier degré en $L$ ou $R$, ou à un monome du second degré en $R$ et l'on en verra l'intérêt plus loin, principalement dans le cas où le calcul économique est à faire pour plus de deux tronçons.

Exceptionnellement, s'il s'agit du calcul économique relatif à deux tronçons seulement, on peut garder la formule $P=a R^{2}+b R$.

Nous voudrions maintenant montrer comment l'on peut traiter ce problème économique pour deux tronçons d'abord, et, dans certains cas, pour $n$.

\section{III. - CAS DE DEUX TRONÇONS \\ $1^{\circ}$ Considérations générales sur la manière de conduire le calcul.}

Soient $x_{1}$ et $x_{2}$ deux paramètres caractérisant chacun des tronçons 1 et 2 . Nos conditions peuvent s'écrire:

- condition de prix minimum :

$$
\begin{array}{r}
P=f\left(x_{1}\right)+g\left(x_{2}\right) \text { minimum, c'est-à-dire } \\
f^{\prime} x_{x_{3}} j x_{1}+g^{\prime} x_{3} \delta x_{2}=0
\end{array}
$$

- condition de perte de charge:

$$
h\left(x_{1}\right)+K\left(x_{2}\right)=B
$$

La différenciation de cette condition donne:

$$
h^{\prime} x_{1} \delta x_{1}+K^{\prime} x_{2} \delta x_{2}=0
$$

Les conditions (3) et (5) correspondent à une variation nulle du coût pour de faibles écarts des paramètres autour de la solution optima, 
écarts choisis de telle façon, si (4) est respecté, que la perte de charge totale reste égale à celle imposée. La condition de compatibilité entre ces deux équations est :

$$
\frac{\partial_{x_{1}}}{\partial_{x_{:}}}=-\frac{g^{\prime}}{f^{\prime}}=-\frac{K^{\prime}}{h^{\prime}}
$$

Dans certains cas simples, cette condition donne une relation très simple entre $x_{1}$ et $x_{2}$; dans d'autres cas, on peut obtenir une relation beaucoup plus complexe. Quoi qu'il en soit, on sait théoriquement trouver $x_{1}$ et $x_{2}$ en combinant cette dernière relation (6) entre $x_{1}$ et $x_{2}$ avec l'équation (4). On obtient ainsi la solution optima.

Sachant exprimer $x_{1}$ en fonction de $x_{2}$ (si la relation (6) peut être rendue explicite), on peut, dans certains cas seulement, transformer (4) de manière à grouper les deưx pertes de charge en une seule, égale à celle d'un tronçon fictif unique ayant les caractéristiques du second et une longueur fictive dont on peut trouver la formule. De même le prix total des deux tronçons est alors celui d'un tronçon fictif unique ayant les caractéristiques du second et la même longueur fictive. On est alors ramené au cas d'un seul tronçon que l'on sait calculer. Cette considération est surtout utile pour la généralisation qui suivra. Quoi qu'il en soit, les relations (4) et (6) donnent toujours le moyen de calculer la dimension caractéristique de chacun des deux tronçons.

a) Si les profils en travers sont susceptibles de se modifier en restant semblables à eux-mêmes, on trouve, en écrivant la condition de compatibilité entre la condition de prix minimum et la condition «différentielle de la perte de charge égale zéro»:

- soit $\left(\frac{R_{1}}{R_{2}}\right)^{6}=c^{\dagger e}$ si le terme conservé dans l'expression du coût est le terme périmètre. - soit $\left(\frac{R_{1}}{R_{2}}\right)^{7}=c^{\text {te }}$ si le terme conservé dans l'expression du coût est le terme section.

- soit $\left(a R_{1}+b\right) R_{1}{ }^{i}=\left(c R_{2}+d\right) R_{12}{ }^{i}$ si la formule du coût comporte les deux termes.

Dans les deux premiers cas, l'élimination de $R_{1}$ entre la formule ci-dessus et celle de la perte de charge totale conduit à l'expression de $R_{2}$ et permet de trouver la longueur fictive dont nous avons parlé.
Dans le troisième, on peut simplement trouver numériquement $R_{1}$ et $R_{2}$.

b) $\mathrm{Si}$, au contraire, on s'impose des profils en travers de hauteur constante, dont seule la largeur est susceptible de varier - il s'agit en général de canaux dans ce cas - on peut toujours garder l'expression complète du coût. II y aura deux cas :

- les canaux sont considérés comme larges (largeurs moyennes $l_{1}$ et $l_{2}$ ). Les rayons hydrau. liques $R_{1}$ et $R_{2}$ sont donc assimilables aux hauteurs $h_{1}$ et $h_{12}$. On sera alors conduit à la rela$\operatorname{tion}\left(\frac{1_{1}}{l_{2}}\right)^{3}=c^{\text {te }}$ et le remplacement de $l_{1}$ par $l_{2}$ dans l'expression de la perte de charge totale donnera $I_{2}$ et aussi l'expression de la lon. gueur fictive d'un tronçon ayant les caractéristiques du second et équivalent à l'ensemble des deux au point de vue des pertes de charge;

- les canaux ne peuvent être considérés comme larges. On sait encore traiter ce cas, mais le plus simple est de poser $R=(1 \vdots)$ h et d'estimer $\Xi$. On est alors ramené au cas précédent ; on le traite et l'on revient, s'il y a lieu, sur l'estimation de $\Xi$.

Traitons intégralement deux exemples:

\section{2" Systrème de deux canaux de coefficients de Chézy différents, dont les prix sont suppo- sés proportionnells aux cubages.}

Pour la recherche de la solution, on suppose que la forme du profil mouillé de ces canaux est donnée et qu'ils ne peuvent varier ici qu'en restant semblables à eux-mêmes. On peut donc les définir par leurs rayons hydrauliques $R_{1}$ et $R_{2}$ et poser, pour les surfaces mouillées et les surfaces brutes (quion pourrait aussi bien supposer distinctes)

$$
S_{1}=a_{1} R_{1}^{2} ; \quad S_{2}=a_{2} R_{2}^{2}
$$

Nos deux conditions prennent, si l'on veut, la forme :

$$
P=K_{1} R_{1}^{2}+K_{2} R_{2}^{2}+c^{t e}=\text { une }
$$

valeur minimum

$$
\text { avec } K_{1 \text { ou } 2}=(f . L \text {. a) } 1 \text { ou } 2
$$

$f_{\text {, et }} f_{2}$ étant les prix unitaires connus et constants dans un domaine limité des variables, $L_{1}$ et $L_{2}$ les longueurs respectives des deux tronçons,

$$
d P=2 K_{1} R_{1} \delta R_{1}+2 K_{2} R_{2} \delta R_{2}=0
$$


D'autre part, la condition de perte de charge s'écrit :

$$
\begin{gathered}
\frac{L_{1} Q^{2}}{C_{1}^{2} R_{1} S_{1}^{2}}+\frac{L_{2} Q^{2}}{C_{2}^{2} R_{2} S_{2}^{2}}=\gamma_{1} R_{1}^{-5}+\gamma_{2} R_{2}^{-5}=B(8) \\
\text { avec } \gamma 1 \text { ou } 2=\left(\frac{L Q^{2}}{C^{2} a^{2}}\right)_{1 \text { ou } 2}
\end{gathered}
$$

qui différenciée devient:

$$
\gamma_{1} R_{1}-\sigma \delta R_{1}+\gamma_{2} R_{2}-6 j R_{2}=0
$$

$\left(7^{\prime}\right)$ et $\left(8^{\prime}\right)$ constituent un système de deux équations linéaires et homogènes en $\partial R_{1}$ et $\partial R_{2}$. La condition de compatibilité est :

$$
\frac{\delta R_{2}}{\delta R_{1}}=-\frac{K_{1} R_{9}}{K_{2} R_{2}}=-\frac{\gamma_{1} R_{1}^{-6}}{\gamma_{2} R_{2}^{-6}} \text { ou } \frac{R_{1}}{R_{2}}=\left(\frac{k_{2}}{K_{1}} \frac{\gamma_{1}}{\gamma_{2}}\right)^{1 / 7}
$$

Remplaçant $K$ et \% par leurs valeurs, il vient, en posant $E_{1 \text { ou } 2}=\left(\frac{1}{f a^{3} C^{2}}\right)_{\text {, ou } 2}$

$$
\frac{R_{1}}{R_{2}}=\left(\frac{f_{2} a_{2}^{3} C_{2}^{2}}{f_{1} a_{1}^{3} C_{1}^{2}}\right)^{1 / 7}=\left(\frac{E_{4}}{E_{2}}\right)^{i / 7}=a_{1}
$$

$E_{1}$ et $E_{2}$ se calculent facilement, donc aussi $a_{1}$.

Faisons en passant la remarque fondamentale pour la généralisation ultérieure que le rapport $\frac{R_{1}}{R_{2}}$ est indépendant de la perte de charge totale que l'on s'est accordée pour le système, indépendant aussi du rapport $\frac{L_{1}}{L_{2}}$.

Tenant compte de (9), (8) peut s'écrire:

$$
Y_{2} R_{2}^{-5}\left[1+\left(\frac{K_{1}}{K_{2}}\right)^{5 / 7}\left(\frac{Y_{1}}{Y_{2}}\right)^{2 / 7}\right]=B
$$

On voit que, si la condition économique est réalisée, la perte de charge de l'ensemble des deux tronçons est égale à celle d'un tronçon unique fictif ayant les caractéristiques du second et la longueur:

$$
L_{2}^{\prime}=L_{2}\left[1+\left(\frac{K_{1}}{K_{2}}\right)^{5 / 7}\left(\frac{\gamma_{1}}{\gamma_{2}}\right)^{2 / 7}\right]
$$

D'autre part, si cette même condition économique est réalisée, l'expression (7) du coût total se met sous la forme:

$$
\begin{aligned}
P & =K_{2} R_{2}^{2}\left[1+\frac{K_{1}}{K_{2}}\left(\frac{K_{2}}{K_{1}}-\frac{\gamma_{1}}{\gamma_{2}}\right)^{2 / 7}\right]+c^{\text {te }} \\
& =K_{2} R_{2}{ }^{2}\left[1+\left(\frac{K_{1}}{K_{2}}\right)^{5 / 7}\left(\frac{\gamma_{1}}{\gamma_{2}}\right)^{2 / 7}\right]+c^{\text {te }}
\end{aligned}
$$

et l'on constate que le coût total est le même que celui d'un tronçon fictif unique ayant les caractéristiques du second et la même longueur fictive $\mathrm{L}_{2}^{\prime}$ que celle définie ci-dessus:

$L_{2}^{\prime}=L_{2}\left[1+\left(\frac{K_{1}}{K_{2}}\right)^{5 / 7}\left(\frac{Y_{4}}{Y_{2}}\right)^{2 / 7}\right]=L_{2}+L_{1}\left(\begin{array}{llll}f_{1}^{5} & a_{1} & C_{1}^{-4} \\ f_{2}^{5} & a_{2} & C_{2}^{-4}\end{array}\right)^{1 / 7}(11)$

$L_{2}^{\prime}$ s'écrit aussi :

$$
L_{2}^{\prime}=L_{2}\left(1+\frac{\gamma_{1}}{\gamma_{2}} a^{-5}\right)=L_{2}+\frac{a_{2}^{2} c_{2}^{2}}{a_{1}^{2} c_{1}^{2}} \frac{L_{1}}{a_{1}^{5}}
$$

On voit donc que, pour le calcul économique, on peut opérer comme suit : on suppose le premier tronçon supprimé et l'ensemble des deux tronçons remplacé par un tronçon fictif unique de mêmes caractéristiques que le second et de longueur $\mathrm{L}_{2}^{\prime}$, fonctionnant sous la perte de charge totale imposée. On commence par calculer $\alpha_{1}$ (formule $9^{\prime}$ ), puis $L^{\prime}$ (formule $1^{\prime} 1^{\prime}$ ). On détermine alors $R_{2}$ du tronçon fictif unique ayant les caractéristiques du second et la longueur $L_{2}^{\prime}$. On en déduit $R_{1}=\alpha_{1} R_{2}$.

La relation $\left(9^{\prime}\right)$ montre que $\frac{R_{1}}{R_{2}}$ doit être d'autant plus petit (donc $\frac{S_{1}}{S_{2}}$ d'autant plus faible, et par conséquent le rapport des pentes $\frac{i_{1}}{i_{2}}$ d'autant plus grand) que $\frac{E_{1}}{E_{2}}$ est plus petit, c'est-à-dire que le premier tronçon est plus coûteux $\left(\mathrm{au} \mathrm{m}^{3}\right)$, de forme plus avantageuse et plus lisse devant le second. 
Tous calculs faits, on trouve :

$$
\begin{aligned}
& \frac{S_{1}}{S_{2}}=\left(\frac{f_{2}}{f_{1}}\right)^{2 / 7}\left(\frac{a_{1}}{a_{2}}\right)^{1 / 7}\left(\frac{C_{2}}{C_{1}}\right)^{4 / 7}(1) \\
& \frac{i_{1}}{i_{2}}=\left(\frac{f_{1}}{f_{2}}\right)^{5 / 7}\left(\frac{a_{1}}{a_{2}}\right)^{1 / 7}\left(\frac{C_{2}}{C_{1}}\right)^{4 / 7}(12)
\end{aligned}
$$

\section{$3^{\circ}$ Autre exemple : système de deux tronçons de conduite forcée (les conduites sont sup- posées constituées par de simples anneaux, sans frettage).}

Admettons que les prix soient proportionnels aux poids, et soit $f_{1}$ et $f_{2}$ les prix du kilo de chacun des tronçons de conduite; $\overline{\text { i) }}$ le poids spécifique du métal : $p_{1}$ et $p_{2}{ }^{-}$les pressions maxima auxquelles chacun des tronçons doit résister ( $y$ compris les surpressions de coups de béliers); $r_{1}$ et $r_{2}$ les taux de travail maximum du métal; $D_{1}$ et $D_{2}$ les diamètres internes des conduites; $e_{1}$ et $e_{2}$ leurs épaisseurs. On aura :

$$
\begin{aligned}
& e_{1}=\frac{p_{1} D_{1}}{2 r_{1}}(15) ; \quad e_{2}=\frac{p_{2} D_{2}}{2 r_{2}} \\
& P=\pi(1)\left(f_{1} L_{1} D_{1} e_{1}+f_{2} L_{2} D_{2} e_{2}\right)+c^{\text {te }}
\end{aligned}
$$

Le report dans (17) de (15) et (16) donne:

$$
\begin{aligned}
& P=\frac{\pi \bar{\omega}}{2}\left(\frac{f_{1} L_{1} P_{1}}{r_{1}} D_{1}^{2}+\frac{t_{2} L_{2} P_{2}}{r_{2}} D_{2}^{2}\right)+c^{1 \theta}= \\
& =K_{1} D_{1}{ }^{2}+K_{2} D_{2}^{2}+c^{t e} \\
& \text { en posant } K_{1 \text { ou } 2}=\frac{\pi_{(1)}^{-}}{2}\left(\frac{f L P}{r}\right)_{1 \text { ou } 2}
\end{aligned}
$$

La condition de coût minimum s'écrit :

$$
K_{1} D_{1} \delta D_{1}+K_{2} D_{2} \delta D_{2}=0
$$

D'autre part, la perte de charge globale des deux tronçons doit être égale à $B$, ce qui s'écrit :

$$
\begin{gathered}
\frac{L_{1} Q^{2}}{\frac{\pi^{2}}{64} C_{1}^{2} O_{1}^{5}}+\frac{L_{2} Q^{2}}{\frac{\pi^{2}}{64} C_{2}^{2} O_{2}^{5}}=\gamma_{1} O_{1}^{-5}+\gamma_{2} D_{2}^{-5}=B \\
\quad \text { avec } \gamma_{\text {1 ou } 2}=\left(\frac{64 \mathrm{LQ}^{2}}{\pi^{2} C^{2}}\right)_{\text {, ou } 2} \text { (22) }
\end{gathered}
$$

La condition d'annulation de la différentielle de la perte de charge s'écrit:

$$
\gamma_{1} D_{1}-6 j D_{1}+\gamma_{2} D_{2}^{-6} j D_{2}=0
$$

La condition de compatibilité entre (20) et (23) est :

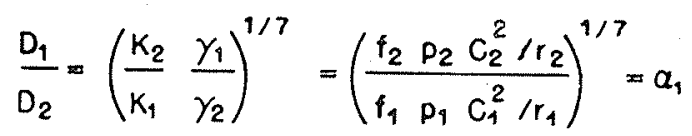

Dans le cas le plus simple, on peut supposer : $f_{2}=f_{1} ; r_{2}=r_{1} ; C_{2}=C_{1} ;$ il reste alors:

$\frac{D_{1}}{D_{2}}=\left(\frac{P_{2}}{P_{1}}\right)^{1 / 7}=\alpha_{1}$

On voit que le diamètre doit être d'autant plus faible que la pression est plus forte. C'est d'ailleurs un fait connu.

Les relations (18), (21) et (24) sont tout à fait semblables aux relations (7), (8) et (9) du $2^{\circ}$. Comme précédemment, on peut donc dire que tout se passe comme si nous avions affaire à un tronçon fictif unique ayant les caractéristiques du second et de longueur :

$$
L_{2}=L_{2}\left(1+\frac{\gamma_{1}}{Y_{2}} a_{1}^{-5}\right)=L_{2}+\frac{L_{1}}{a^{5}} \frac{C_{2}^{2}}{C_{1}^{2}}
$$

En résumé, le calcul économique d'un ensemble de deux tronçons de conduite forcée se conduira comme suit :

$$
\text { - calcul à l'aide de (24) de } \alpha_{1}=\frac{D_{1}}{D_{2}} \text {; }
$$

- calcul par la relation (25) de la longueur $L^{\prime}$ : du tronçon fictif ayant les caractéristiques $p$, $D, r, f$, du second et équivalent à l'ensemble des deux, du point de vue des pertes de charge et du point de vue du prix;

- calcul de $D_{2}$ comme pour un tronçon unique de longueur $\mathrm{L}_{2}^{\prime}$;

$$
\text { - retour à } D_{1}=\alpha_{1} D_{2} \text {. }
$$

Il se peut qu'ayant ainsi obtenu une paire de valeurs pour $D_{1}$ et $D_{2}$ on constate qu'elles ne correspondent pas à l'idée qu'on s'en faisait (désir d'obtenir des nombres ronds, suffisamment différents, etc...) et que l'on veuille agir sur elles en modifiant la position de la sépara- 
tion entre les deux tronçons. De ce fait, on modifie $\frac{P_{2}}{P_{1}}$ donc $\alpha=\frac{D_{1}}{D_{2}}$ et $L_{2}^{\prime}$ et l'on obtient deux nouvelles valeurs pour $D_{1}$ et $D_{2}$.

\section{IV. - CAS DE PLUSIEURS TRONÇONS}

Nous allons montrer, sans utiliser les mathématiques, qui permettraient aussi de le faire aisément, que l'on peut généraliser la méthode cidessus au cas de $n$ tronçons, à condition qu'elle soit applicable à deux tronçons consécutifs quelconques pris parmi ces $\mathrm{n}$.

Supposons le problème résolu et nos variables caractérisant les dimensions choisies précisément de manière à satisfaire à nos deux conditions, la condition de perte de charge -totale imposée et la condition de prix minimum. Dire que ces conditions sont satisfaites, c'est dire que l'on peut, sans changer le prix, faire notamment varier, d'une quantité très petite, deux des̊ paramètres - par exemple les deux premiers - à condition de lier leurs variations de manière que la perte de charge totale ne change pas. Donc le prix total et la perte de charge totale ne changeant pas, et rien n'étant changé par ailleurs pour aucun tronçon en dehors des deux premiers, la somme des pertes de charge de ces deux tronçons et la somme de leurs prix ne changent pas. Cela veut dire que l'on se trouve aussi respecter la condition économique relative aux deux premiers tronçons considérés comme seuls. Or, cette condition est, dans bien des cas, on l'a vu dans nos exemples, indépendante de la charge; elle s'exprime par un rapport entre les deux variables caractéristiques, rapport que donnent les calculs du paragraphe 111 .

On peut donc, pour respecter la condition économique relative aux deux premiers tronçons, poser :

$$
\mathrm{R}_{1}=\alpha_{1} \mathrm{R}_{2} \text { ou } \mathrm{D}_{1}=\alpha_{1} \mathrm{D}_{2}
$$

( $\%$, est donné dans nos exemples par (9) ou (24), puis remplacer les deux premiers tronçons par un tronçon fictif ayant les caractéristiques du second, mais de longueur fictive $L^{\prime}$ (donnée dans nos exemples par les relations (11) et (25).

De la même façon on peut remplacer l'ensemble des tronçons $L_{2}^{\prime}$ et $L_{3}$ par un tronçon fictif unique $L_{3}^{\prime}$ représentant l'ensemble des tronçons $1,2,3$, etc..., et de proche en proche on réduit le système à un tronçon fictif unique ayant les caractéristiques du dernier et une longueur $L^{\prime}$. déterminée par applications successives d'une formule et sans tâtonnements, dont on calcule alors la dimension caractéristique et la pente, s'il y a lieu, de manière à satisfaire à la perte de charge totale imposée. Les chiffres trouvés sont valables pour le dernier tronçon et l'on remonte de proche en proche au premier par la succession des formules:

$$
R_{n-1}=\alpha_{n-1} R_{n} ; \ldots \ldots R_{1}=\alpha_{1} R_{2}
$$

Il se peut que la vue du tableau des résultats incite à grouper certains tronçons, à en rallonger ou en raccourcir d'autres, etc., de manière à avoir une variation plus satisfaisante du paramètre choisi. Seule l'expérience dira dans quel sens on doit agir pour obtenir ce résultat.

II s'agit là d'une méthode relativement rapide et sûre, applicable à condition de savoir évaluer l'influence de la section des canaux (ou exceptionnellement de leur périmètre) ou du poids des conduites sur leur coût, et dans la mesure où l'on peut donner au prix une expression qui comporte un seul monôme contenant la variable choisie.

Le lecteur verra de lui-même si la méthode est applicable à son cas particulier; il suffit, pour cela, que chaque tronçon puisse être incorporé au tronçon suivant par la considération d'une longueur fictive de ce dernier. Cela nécessite, croyons-nous, que, d'un tronçon au suivant, les expressions du prix et celles de la perte de charge aient respectivement la même forme. Cependant, si le système comporte seulement deux groupes de tronçons hétérogènes entre eux, on doit pouvoir encore le calculer en traitant séparément ces deux groupes (après avoir introduit une variable supplémentaire, la perte de charge totale de l'un d'eux) et en se ramenant alors à deux tronçons fictifs, pour lesquels on sait trouver la solution économique dans tous les cas.

Cette façon d'opérer serait encore valable si les tronçons homogènes entre eux n'étaient pas consécutifs, mais alternaient d'une façon quelconque avec ceux de l'autre groupe.

Ayant trouvé certains résultats, c'est-à-dire certaines proportions dans les dimensions caractérisant les différents tronçons, le lecteur ne devra pas se croire obligé de les respecter rigoureusement. II ne faut pas se dissimuler en effet que les calculs sont approximatifs dans la mesure 
même où les hypothèses sont incertaines. D'autres chiffres assez voisins pris à la base des calculs auraient conduit à des résultats un peu différents. C'est ainsi que règne une incertitude assez forte sur la variation des prix avec les dimensions, ou sur les coefficients de Chézy. qu'on ne peut jamais connaitre rigoureusement et que l'on est contraint d'estimer au début des calculs, avant de connaître les dimensions auxquelles on sera conduit et dont ils dépendent quelque peu.

Une extension graphique de cette méthode sera donnée dans un prochain numéro.

\section{BIBLIOCRAPHIE SOMMAIRE}

- PACLIARO: Dimensions économiques d'une conduite forcee La Elettrotecnica, 1919.

- SANTO RINI : La solution zénérale du problème de la détermination des dimensions économiques maximum d"une conduite forcée métallique et son application aux calculs pratiques. Lio Houille Blanche, 1925.

- CATAN1: Tecnica ed economia nelle condotte forzate a dia metri variabili. La Houille Blanche, 1933.

- VERONESE : Criteri di economia per il calcolo di condotte L'Ingegnere, mars 1942

- GUIDO FERRO : Minimum de déblais et minimum de dépenses dans les canaux. Energia Elettrica, juillet 1932.

- MARzOlo : Sur le dimensionnement économique des camalu et conduites dans les centrales hydroélectriques. Energia Elettrica. février 1940.

STRECTER: Economical canal
ASCE, vol. 110 , page 2242, 1943 .

- UCO BELLOMETTI: Défermination des diamètres variables qui rendent minimum le poids d'une conduite forcée. La Houille Blanche, septembre-octobre 1947 .

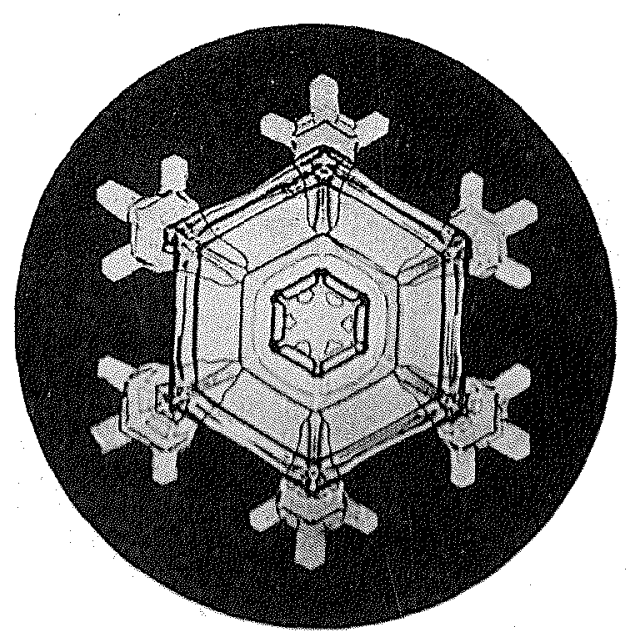

